\title{
First Workshop on \\ Artificial Intelligence in Education to Support the Social Inclusion of Communities (AIEDSIC)
}

\author{
Fábio N. Akhras ${ }^{1, *}$ and Paul Brna ${ }^{2, *}$ \\ ${ }^{1}$ Renato Archer Center of Information Technology, São Paulo, Brazil \\ fabio.akhras@cti.gov.br \\ ${ }^{2}$ School of Informatics, The University of Edinburgh, Scotland, UK \\ paulbrna@mac.com
}

\begin{abstract}
Summary. About 20 years ago, in a paper entitled "Computational Mathetics: the Missing Link of Artificial Intelligence in Education", John Self argued that AI in Education has missed its connection with formal AI, its theoretical side. Some people argued that this was necessary so that AI in Education (AIED) could be able to deliver real world applications. However, in the real world, half of the population lives with less than 3 dollars a day with many socially excluded from education, health and other basic services. Social inclusion seeks to address the needs of this population, mostly living in underdeveloped countries, and also combat factors that are socially problematic in developed countries such as poor educational attainment, unemployment, poor health/special needs, low income, crime and poor housing/local environment.

The AI in Education community has spent more than 30 years researching the design of adaptive technologies to support learning. However, the issue of supporting social inclusion has never been directly addressed. Has AI in Education also missed an important connection with the real world? We argue that AI in Education systems have a challenging role to play in helping to transform communities but we also accept that much has to be done to establish the ways in which work on AI in Education supports such activities indirectly, and to determine what future work needs to be done.

The European Union made 2010 the European Year For Combating Poverty and Social Exclusion. The key objectives were to improve public awareness and commitment at the political level to fight poverty and social exclusion while some key challenges are: to eradicate child poverty by breaking the vicious circle of intergenerational inheritance, to promote the active inclusion in the society and the labour market of the most vulnerable groups, to overcome discrimination and increase the integration of people with disabilities, ethnic minorities and immigrants and other vulnerable groups. We can start by focusing on AIED's capacity to support these aims. Therefore, the main purpose of this workshop is to identify and discuss the challenges that arise in addressing issues of supporting the social inclusion of communities in the context of $\mathrm{AI}$ in Education research and lay the groundwork for future workshops in this area.
\end{abstract}

Programme Committee: Robert Aiken (Temple Univ., USA), Nicolas Van Labeke (Univ. of Nottingham), Rose Luckin (Inst. of Education, UK), Jack Mostow (Carnegie Mellon Univ., USA), Gilda Olinto (Brazilian Inst. of Information on Science and Technology, IBICT, Brazil), Natasha Queiroz (Federal Univ. of Paraiba, UFPB, Brazil), Rafael Morales (Univ. of Guadalajara, Mexico).

* Workshop Co-Chairs. 